\title{
Listening to student voice-understanding student and faculty experience at two UK graduate entry programmes
}

\author{
M. Abdulhadi Alagha ${ }^{1,2^{*}}$ and Linda Jones ${ }^{2}$
}

\begin{abstract}
Context: Shortage of physicians in the UK has been a long-standing issue. Graduate entry medicine (GEM) may offer a second point of entry for potential doctors. However, the challenges of developing and implementing these programmes are still unrecognised. This small-scale study aimed to briefly explore the opportunities and challenges facing students at two UK GEM programmes.

Methods: Two case studies were conducted at Imperial College and Scotland's GEM (ScotGEM) and used a triangulated qualitative approach via semi-structured and elite interviews. Data analysis, informed by grounded theory, applied thematic and force-field analysis in an empirical approach to generate evidence and instrumental interpretations for Higher Education Institutions.

Results: Although GEM forms an opportunity for graduates to enter medicine, the different drivers of each programme were key in determining entry requirements and challenges experienced by postgraduates. Three key dilemmas seem to influence the experiences of learners in GEM programmes: (a) postgraduate identity and the everchanging sense-of-self; (b)self-directed and self-regulated learning skills, and (c) servicescape, management and marketing concepts.

Conclusions: Graduate entry programmes may support policy makers and faculty to fill the workforce gap of healthcare professionals. However, their successful implementation requires careful considerations to the needs of graduates to harness their creativity, resilience and professional development as future healthcare workers.
\end{abstract}

Keywords: Graduate entry, GEM, Sense-of-self, SDL, Servicescape

\section{Introduction}

Admission policies for prospective medical students in the UK have been a subject of debates raging for centuries $[1,2]$. Traditionally, medicine in the UK has been a taught undergraduate degree over $5 \mathrm{y}$, with learners joining the programmes soon after completing their high school education. However, the shortage in healthcare workforce led policy makers to consider widening access

\footnotetext{
* Correspondence: h.alagha@imperial.ac.uk

'Institute of Global Health Innovation, Department of Surgery and Cancer, Faculty of Medicine, Imperial College London, London SW7 2AZ, England ${ }^{2}$ Centre for Medical Education, Faculty of Medicine, University of Dundee, Dundee DD2 4BF, Scotland
}

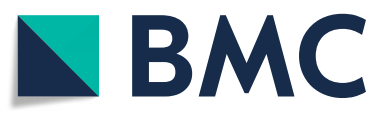

(C) The Author(s). 2021 Open Access This article is licensed under a Creative Commons Attribution 4.0 International License, which permits use, sharing, adaptation, distribution and reproduction in any medium or format, as long as you give appropriate credit to the original author(s) and the source, provide a link to the Creative Commons licence, and indicate if changes were made. The images or other third party material in this article are included in the article's Creative Commons licence, unless indicated otherwise in a credit line to the material. If material is not included in the article's Creative Commons licence and your intended use is not permitted by statutory regulation or exceeds the permitted use, you will need to obtain permission directly from the copyright holder. To view a copy of this licence, visit http://creativecommons.org/licenses/by/4.0/ The Creative Commons Public Domain Dedication waiver (http://creativecommons.org/publicdomain/zero/1.0/) applies to the data made available in this article, unless otherwise stated in a credit line to the data. to the medical profession for mature and graduate students [3]. Graduate entry medical (GEM) programmes are usually four to $5 \mathrm{y}$ in duration targeting graduate learners [4]. The first GEM programme in the United Kingdom was established in year 2000 and have steadily increased in popularity since then $[3,5]$.

Medical education in the United States of America has been specifically designed for graduate learners who have completed at least a four-year Bachelor of Science degree [6]. The British and American models of medical education have been the subject of much comparison regarding their effectiveness [7]. The differences between 
undergraduate (UG) and graduate learners are widely described in the literature $[8,9]$. For example, the superior academic performance of GEM students (being postgraduate), as compared to their UG peers, is thought to be attributed to factors such as age, maturity, self-regulation, motivation, class engagement and prior work experiences [9-11]. Trueman and Hartley [12] attributed this to better time and stress management skills that mature learners bring and is in harmony with studies reporting that high anxiety levels significantly hinder the performance of UG students [13, 14].

Sanford's challenge and support theory [15] showed that for an optimal learning experience and personal growth, challenges encountered must match the support provided. A practical interpretation of this theory implies that graduate students confront different challenges from those of undergrads and therefore, both the type and degree of support provided for each of these groups should be student-specific [16].

Shotter and Gergen [17] challenged notions of identity being fixed arguing that it develops dynamically through interactions with others [18]. Learners' sense-of-self therefore undergoes a process of continuous transformation during their educational experience [19] and they need to be able to bring and build selves in line with the learning outcomes [20].

Despite the consistency and integrated nature of educational theories with graduate learning, the literature seems to lack sufficient explicit strategic knowledge of how to optimise curricula design and graduates' learning experiences. This small-scale study sought to understand the challenges and opportunities encountered by graduate learners through reconciling the views of stakeholders in two GEM programmes of different characteristics, as highlighted below.

\section{Methods}

An iterative qualitative case study approach used semistructured and elite interviews to explore three research questions:

- What do stakeholders, in two graduate-entry programmes, perceive to be the challenges and opportunities for graduate entrants into medicine?

- What strategies do stakeholders find helpful in managing these challenges?

- What ideas and/or recommendations are needed to maintain or enhance the existing design and delivery of the programmes?

Data collection and analysis were informed by the principles of grounded theory [21, 22]. Using force-field and thematic analyses, we sought to explore the opportunities and challenges of students and elite faculty stakeholders to construct evidence for possible enhancement of design and delivery of GEM programmes. The results are instrumental recommendations generated through interactions with students in the semi-structured interviews and programme leaders in the elite interviews.

\section{Setting and Programme differences}

The case studies were conducted at Imperial College London (ICL) in England and the ScotGEM programme, a collaboration between the Universities of St. Andrew's and Dundee in Scotland. Selection of medical schools followed an opportunistic and purposive sampling strategy [23] wherein MAA had a dual student identity at both schools yet was an outsider researcher in the context of GEM.

ScotGEM is a four-year programme, established by the Scottish government as a specialist effort to meet the growing needs of remote and rural generalist physicians in National Healthcare System (NHS) Scotland [24]. ICL's programme, which at the time of study was in suspension for prospective students [25], is a five-year programme aimed to develop physicians with a keen interest in academia and research [26]. It is noteworthy that while ICL's programme is aimed at science degree holders, ScotGEM accepts graduates of any field. On average, ICL and ScotGEM programmes had the capacity to accommodate up to 20 and 50 students per academic year, respectively.

\section{Participants}

We conducted purposive sampling for elite interviews with faculty staff and student representatives [23]. Student participants were recruited randomly through short announcements as well as recruitment posters, emails and leaflets, followed by a snowball research technique $[27,28]$. Semi-structured (SS) interviewees were grouped randomly whilst elite interviews were conducted on a one-to-one basis. We composed three stakeholder-specific groups consisting of faculty (E), student representative (SR) and students (S); ScotGEM (faculty-elite: 2, SRelite: 1, students-SS: 11 in two groups), ICL (faculty-elite: 1, SR-elite: 1, students-SS: 2 in one). Written informed consent was obtained from all participants. No incentives were offered for participating. Table 1 classifies participants at ScotGEM and ICL. All methods were carried out in accordance with relevant guidelines and regulations.

\section{Data collection}

ScotGEM and ICL interviews were conducted, recorded and transcribed between August and October 2019. Hour long semi-structured non-directive interviews (SSI) and elite interviews of about $45 \mathrm{~min}$. Personal 
Table 1 ScotGEM and ICL interviews

\begin{tabular}{lll}
\hline Elite Interviews & \multicolumn{1}{c}{ ScotGEM } & Semi-structured interviews \\
ScotGEM Programme Director (E1) & One-to-one interview & Group 1: G1S1, G1S2, G1S3, G1S4, G1S5, G1S6 \\
Senior Educator/Leader of GEM (E2) & Student Representative (SR) & Group 2: G2S1, G2S2, G2S3, G2S4, G2S5 \\
& Imperial College GEM & \\
Senior Educator/Leader of GEM (ICL-E1) & Student Representative (ICL-SR) & Group: ICLS1, ICLS2 \\
\hline
\end{tabular}

identifiers were anonymised while contextual identifiers in individuals' stories remained to ensure robustness of the data [29]. Given the possible contextual identifying factors, faculty elite interviewees were given the opportunity to edit and sign off the data related to their interview. Although SRs were purposively sampled as elite interviewees, they were not given this privilege however some of their findings were included in students' voice not to obscure meaning but to ensure anonymity.

\section{Data analysis}

Cycles of simultaneous data collection and analyses took place in an iterative process until a level of theoretical saturation was reached [21]. A blend of inductive and deductive analytical approaches by MAA and LJ allowed comparison and triangulation between students and faculty [22] Open codes were cross-checked and disparities were discussed until consensus was reached. Codes were subsequently clustered into conceptual narrative themes to drive theoretical model and conceptualise the dilemmas facing GEM learners [21].

The study encouraged ongoing broad openness and authenticity for reflexive accounts "reflexivity" to explore any existing "conceptual leaps" and to articulate the influences shaping the research [30]. Applying principles of grounded theory, we adopted an emergent approach with constant comparisons from conceptualisation to writing-up in order to explore the similarities and differences between the groups [22]. Similarly, the 'so what' factor contributed to identifying and comprehending the social phenomenon as it emerged [31].

Following data gathering and analyses, a secondary literature review was undertaken of emerged key concepts. This iterative cycle and integrated approach of literature review allowed a thorough blend of perspectives and principles required to develop a holistic understanding [32].

\section{Results}

Although GEM forms an opportunity to embrace the healthcare profession, the challenges facing GEM learners were perceived differently in each case studied and the drivers of each programme influenced their nature.

It comes without saying that comparison and generalisation of case studies is difficult. However, it was possible to compare "apples and pears" using cross-case analyses and broad descriptors [33]. We now present and discuss our findings from both case studies together to illuminate recommendations through different lenses..

\section{Drivers, motivations and expectations}

The drivers of each programme seem to be key in shaping entry requirements and challenges experienced by learners. Based on Lewin's Force Field Analysis (FFA) [34], Fig. 1 illustrates the two sets of forces influencing stakeholders' behaviours.

ScotGEM, financed by the Scottish government to address the skill gap of qualified doctors, emphasised the political and geographical factors associated with its' remote and rural $(R \& R)$ agenda:

"ScotGEM has got this clear vision that was kind of given to us by our funders ... we are kind of commissioned course; they (funders-Scottish Government) said we're interested in generalism ... and rural"

"We are all coalesced around about the primary objective, which is to serve the communities of Scotland in particular".

E1 felt that although ScotGEM has been designed and delivered by two universities, its vision was policy and workforce driven, which may be different if the two universities were to collaborate on establishing a GEM programme.

On the others hand, ICL's GEM programme focused on developing physicians with a keen interest in academia and research:

"So the government requires us to train generalists because that's what the national health service wants. Imperial college is a science and research focused university and we spend a lot of time teaching our medical students how to do research and be research focused and evidence-based".

(ICL-E1)

In line with these findings, the types of forces facing GEM stakeholders were categorised in four ways: 


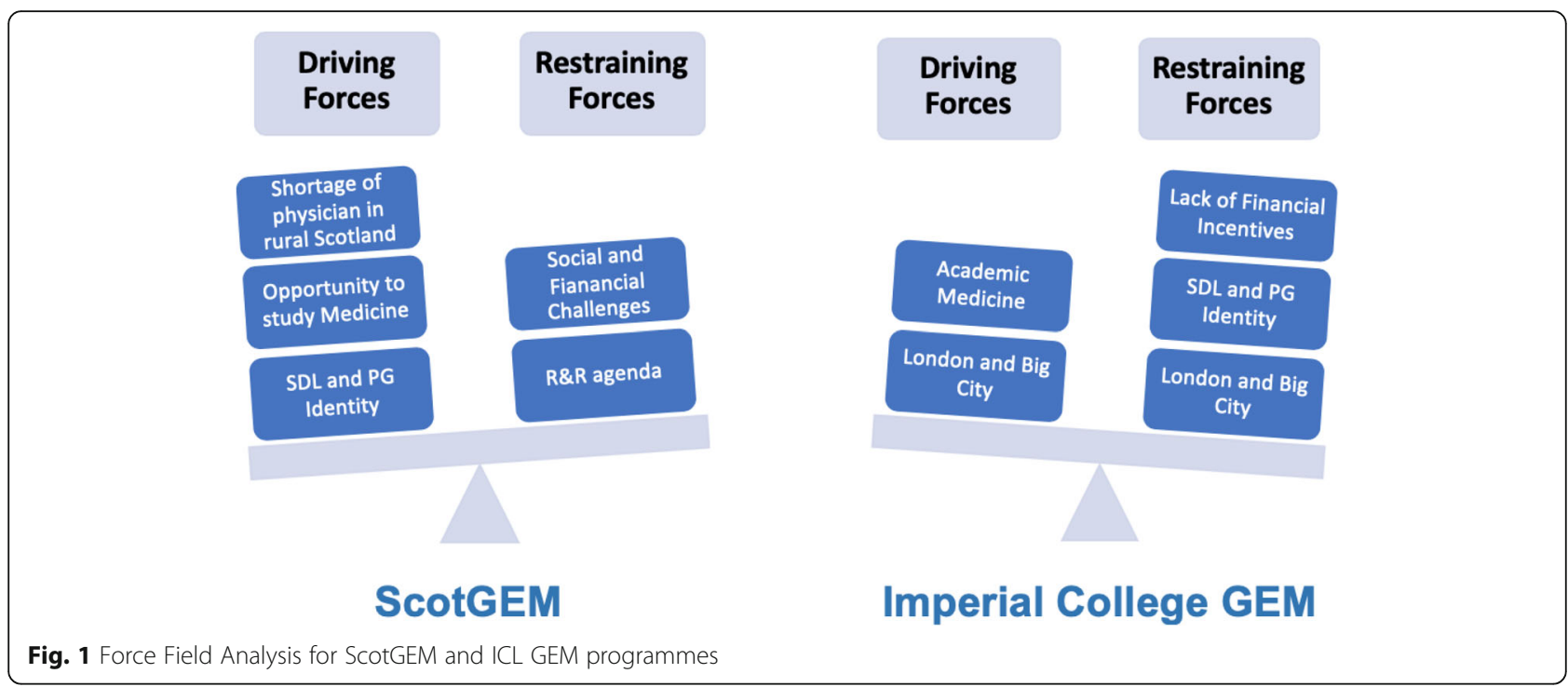

behavioural, political, geographical and learner-specific. Table 2 shows the types of forces to consider at both medical schools.

\section{Sense-of-self, professional identity and self-directedness}

The two case studies highlighted the way sense-of-self for GEM learners is supported and managed differently. It seemed that the experience of ICL students of being taught amidst their UG peers, generated some frustration regarding their postgraduate identity and SDL skills:

"I think it can be quite demoralising ... there's no recognition of the fact that these people have PhDs and have high levels of degrees and there's no recognition that there is a level of higher scientific education with these graduate-entry students versus undergraduates who essentially have come from $A$ levels".

(ICL-S1) in agreement with ICL-S2

"I have a master's, I've worked, I feel mature ... it's not about being surrounded by younger cohort but I guess just my attitude is different. I want to be surrounded by like-minded people and so coming to Imperial to find out that ... it's essentially an undergraduate course hidden in the need of graduate medicine ... so that's the mindset barrier, I would have to commit not just to the money, but to almost applying to an undergraduate course despite my previous background".

(ICL-S2)

Whilst that sense of identity as postgraduates was less problematic when taught as a separate cohort as with ScotGEM:

"I think as a graduate learner, I am a fan of sorting everything for myself. I don't want to join a graduate-entry course and then feel too much like an undergraduate, even though technically medicine is (well at least here) still an undergraduate program. I wouldn't want to go ahead and having studied and learned how to be a student and done two qualifications to then be in lectures nine to five to being spoon-fed information"

$(S R)$

\section{Management, marketing and servicescape}

Student choice of university to study GEM was addressed in both case studies. Although ScotGEM learners found the newly established programme as an opportunity to get into medicine, they seemed to be more rationally driven by its $R \& R$ agenda. However, the unreliable and infrequent public transportation in rural

Table 2 Types of Forces facing GEM Stakeholders

\begin{tabular}{llll}
\hline Types of Forces & & & \\
\hline Prior Educational Knowledge required & SDL & Research Involvement & Part-time Work \\
Policy Makers' Vision & Financial Resources & Market and NHS Needs & Focus of GEM programme \\
Location of Medical School & Big City & Remote and Rural Settings & Transportation \\
Social and Family Arrangements & Support Services & Adult Learning Principles applied & Flexibility \\
\hline
\end{tabular}


Scotland makes it challenging to reach clinical practices on time:

"There was a specific question online ... will you need a car? They said No, no. All the places have buses but..."

(G1S1)

"for our placements in first year have been quite far away. If you were going to get the bus or the train, it would take you an hour and a half to get there"

(G1S2)

A few students expressed worries about leaving their partners, children and pets for several weeks. One frustrated student said "if you're moving for five weeks, it's like, I'm not gonna move my cat for five weeks each time I have to go somewhere".

(G2S2)

E2 highlighted that both UG and GEM learners face different set of social challenges that they need to address. For example, although GEM might pose additional responsibilities related to family, children, caring for parents... et cetera, they are usually more organised about their ways of living, their confidence regarding planning and being comfortable managing aspects of independent living that UG might find challenging:

"I think life is so complex now, you can make too many generalisations about the demands being similar or different for these groups".

The need to retain these future clinicians led to the development of a strategic recruitment process as explained by E1 "so our recruitment strategy was designed around trying to preference people who were at least willing to sign up for that (to work as generalist physicians in depleted areas)". That said, entry requirement to ScotGEM such as entry examinations (GAMSAT, UKCAT) and advanced/university-level chemistry is believed to be a deterring factor for the people who are particularly interested in serving remote areas:

"So we set some hurdles that are probably unnecessary, may be deterring people from applying to ScotGEM who are exactly the people we wanted because of their backgrounds and where they're interested in working in longer term"

(E1)

In contrast, ICL students were arguably more influenced by emotional factors and these included: career prospects, opportunities to be involved in research and the lifestyle in a big city like London. However, being classified as an undergraduate course resulted in financial barriers, which in turn led to social difficulties, seemed to be ICL's key challenges:

"One of the biggest things is the funding isn't sufficient enough ... and that takes away from, just having time to have a personal life, time to even come into university for like lectures and things ... So it's quite a big challenge finding time to be with your family, finding time to be with your partners".

(ICL-SR) view was echoed by ICL-E1, ICL-S1, ICL-S2

\section{Discussion}

Our findings suggest that whilst the two GEM programmes seem to meet the needs of healthcare workforce recruitment by offering another point of entry for potential doctors, the student experience suggests some further adaption to graduates' needs may enhance successful implementation. Our findings furthermore suggest that enhancement of GEM programmes requires the management of three key dilemmas (Fig. 2). Firstly, postgraduateness and sense-of-self needs to be deliberately recognised and valued. Secondly, the impact of geographical, social and emotional dimensions of learning environments on graduates requires careful consideration and balance. Finally, students' previous degrees and abilities to self-regulate and self-direct their learning may need to be adequately acknowledged.

Our understanding of these three dilemmas was enhanced through a secondary literature review of emerged themes. Self-identity is defined as the way an individual perceives their thoughts, values, believes, traits and purpose within a culture [35]. On the other hand, consumption behaviour and marketing theories [36] accept the provision of education as a complex service and appreciate its service environment (also known as Servicescape) to shape entry requirements and increase "buy-in" [37]. Self-directedness, which is part of adult education theory, is the ability to regulate own behaviour to adapt to the needs of personal goals [38]. Despite SDL's frequent use in educational discourses, several authors

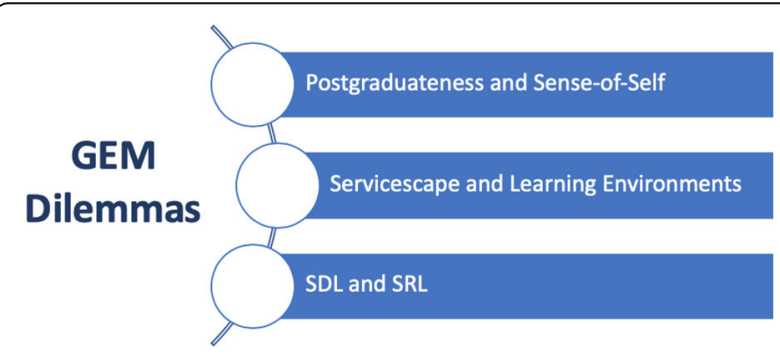

Fig. 2 Key dilemmas of GEM learners 
highlighted that the concepts of SDL and SRL are rather intangible $[39,40]$; SDL is thought to be a broader construct encompassing SRL, which has less freedom to manage own learning initiatives [41].

Central to understanding the challenges facing GEM learners and prosperity of programmes is the explicit articulation of the vision and needs of each party; policy makers, faculty staff and students. In line with previously conceptualised professional identity formation [42, 43], our study recommends that programme designers may need to consider ways to enhance postgraduateness through designing bespoke GEM programmes and customised professional development initiatives [44]. For graduates who are part of a bigger cohort of UG and GE learners, this may be through deliberate recognition of students' graduateness and the development of strategies that value and utilise the everchanging sense-of-self [45, 46]. By the same token, prospective students seem to appreciate explicit signposting to potential challenges facing them to facilitate their informed choice to apply for a specific course [47].

With regards to servicescape concept and recruitment strategies [37], our findings draw attention to the importance of understanding how learning environments affects GEM learners to make programmes more attractive for prospective applicants. Vygotsky [48] recognised the importance of interpersonal and personality attributes of learners in educational contexts and advised that care and sensitivity need to be given to accommodate learners' preferences [49]. The different challenges facing mature learners, such as family or even pet care; $R \& R$ transportation; and financial/living cost difficulties, in comparison to their undergraduate peers [16] prompt the need for bespoke support services to get a maximum output for the input that they give. This may be through working closely with learners to develop strategies and provide provisions for financial incentives to support students (e.g. purchase a car or assist with living costs) [49], part-time work, flexible learning and logistics support services [50-52].

Previous research findings have shown that graduates need to be able to bring their own self and experiences to regulate their learning with appropriate guidance from faculty [20]. Inclination towards SDL may vary on the continuum of learning $[53,54]$. The closer a curriculum is to SDL, the more creative, successful, confident and resilient learners may be $[55,56]$. We feel that our findings provide empirical evidence for this issue conveyed by Poole [20]. Graduates clearly expressed a desire to recognise prior degrees and abilities to self-regulate their learning needs. Based on our findings, an explicit shift towards a more self-directed approach on the SDL continuum may help GEM students to harness their creativity and enhance their resilience and sense-of-self as graduates within the larger medical school cohort and/ or as a graduate undertaking further undergraduate study [54].

\section{Implications}

Findings from our study highlight elements in curricula design that may enhance the learning experiences of graduate learners. Graduates bring their own personal and professional experiences to educational contexts and may help provide an additional layer of peer support and SDL [57]. Therefore, active appreciation of their graduateness, endorsing their postgraduate sense-of-self and capacity for self-directedness could be key to optimising curricula and their professional development. Stakeholders of existing programmes believe GEM is working well. For instance, graduate medicine affords a transparent path to meet the shortage of doctors [3], might it possible that other allied healthcare professionals such as dentists, physiotherapist and occupational therapist, benefit form graduate-entry programmes to meet their own needs?

\section{Limitations and future directions}

There are few limitations to report. Although the case study design was not aimed at generalising our findings to other educational contexts, our two-centre study triangulated the experiences of students and faculty leaders at two institutions in England and Scotland which have dissimilar drivers. We hope our constructivist empirical approach was better judged by what it conveyed in terms of plot, participants and place while convincing readers of its representativeness [58]. Though it may not be generalisable, case study research comprises of systematic data collection and analyses hence findings may be relevant to other contexts and situations [59]. Furthermore, the smaller number of interviews at ICL may have limited readers' confidence in reaching a saturation point. However, given the number of students at ICL GEM per academic year (average 20) as well as researchers' perception of no new emerging information in data analysis, may ensure an adequate sample size [21]. Future research involving multiple sites and programmes nationally and internationally, would be beneficial to understand differences in opportunities and challenges available for graduates. Finally, our study only focused on student and staff perspectives. Although FFA provides a personalised product and is seen as a people process [60], it often requires the commitment of all stakeholders to get the recommendations acted upon. Therefore, governmental, political and patient cultures play an active part in this change management initiative and are likely to have a significant impact on the successful execution [61]. 


\section{Conclusions}

Stakeholders appear to be valuing the experiences of GEM. Most recognised the importance of continued commitment to enhance the experience of individuals and institutions, in ways which endorse the political or market drivers. Graduate entry programmes comprise of individual postgraduate learners choosing to transition back to an undergraduate level. Our study suggests that programme providers could support these unique students by strategies which acknowledge and maintain their postgraduate identities, recognise and address their needs as mature students, utilise the graduate skills they have already acquired within mixed peer groups and to build capacities for SDL. Future research on how to best manage and value these key dilemmas in GE education is needed to influence the prosperity of programmes, improve the learning of future healthcare workers and arguably the safety of their patients.

\section{Acknowledgements}

We thank the staff and students of Imperial College London and ScotGEM who helped with the recruitment, and the interviewees who participated in our study.

\section{Authors' contributions}

MAA, as part of his Masters dissertation, was responsible for conceiving and conducting this study, data analysis and the development of the manuscript. $\downarrow$ supervised the conception of the study, the data analysis and manuscript development. The authors read and approved the final manuscript.

\section{Authors' information}

M. Abdulhadi Alagha MD, MSc, MMEd is a Teaching Fellow at the Institute of Global Health Innovation of Imperial College London.

Linda Jones PhD is a Senior Lecturer in Medical Education at the Centre for Medical Education, University of Dundee.

\section{Funding}

No funding was received for this project.

\section{Availability of data and materials}

The datasets used and/or analysed during the current study available from the corresponding author on reasonable request.

\section{Declarations}

Competing interests (same as provided on the submission system) The authors report no conflict of interests.

\section{Ethics approval and consent to participate}

This study was approved by the School of Medicine and School of Life Sciences Research Ethics Committee at the University of Dundee (project no. 19/32). Site approvals were obtained from the University Teaching and Research Ethics Committee (UTREC) at the University of St. Andrew's and from both the Joint Research Compliance Office (JRCO) and Medical Education Ethics Committee (MEEC) at Imperial College London. Written informed consents were obtained from all participants. All methods were carried out following relevant guidelines and regulations.
Received: 16 January 2021 Accepted: 25 March 2021

Published online: 05 April 2021

\section{References}

1. Searle J. Graduate-entry medicine: what it is and what it isn't. Med Educ. 2004;38(11):1130-2 https://doi.org/10.1111/j.1365-2929.2004.01984.x.

2. Wood D. Medical school selection - fair or unfair? Med Educ. 1999;33:399401.

3. Powis D, Hamilton J, Gordon J. Are graduate-entry programmes the answer to recruiting and selecting tomorrow's doctors? Med Educ. 2004;38(11): 1147-53 https://doi.org/10.1111/j.1365-2929.2004.01986.x

4. Calvert MJ, Ross NM, Freemantle N, Xu Y, Zvauya R, Parle J. Examination performance of graduate entry medical students compared with mainstream students. J R Soc Med. 2009;102(10):425-30 https://doi.org/1 0.1258/jrsm.2009.090121.

5. Knight J, Stead AP, Geyton TO. Comparing the academic performance of graduate-entry and undergraduate medical students at a UK medical school. Educ Health. 2017;30:75-8.

6. Nara N, Suzuki T, Tohda S. The current medical education system in the world. J Med Dent Sci. 2011:58(2):79-83.

7. Marsden JS. An insider's view of the American and UK medical systems. Br J Gen Pract. 2006:56(522):60-2

8. Choudhury I. A comparative analysis of performance by graduate and undergraduate students in an MEP course. Seattle: 122nd Annual Conference of the American Society for Engineering Education; 2015.

9. Duggan EM, O'Tuathaigh CMPO, Horgan M, O'Flynn S. Enhanced research assessment performance in graduate vs. undergraduate-entry medical students: implications for recruitment into academic medicine. QJM. 2014; 107(9):735-41.

10. Friday E, Friday-Stroud SS, Green AL, Hill AY. A multi-semester comparison of student performance between multiple traditional and online sections of two management courses. J Behav Appl Manag. 2006;8(1):66-81.

11. Nerad M, Miller DS. Increasing student retention in graduate and professional programs. In: Haworth JG, editor. Assessing graduate and professional education: current realities, future prospects (new directions for institutional research), vol. 42. San Francisco: Jossey-bass; 1996. p. 61-76.

12. Trueman M, Hartley J. A comparison between time-management skills and academic performance of mature and traditional-entry university students. High Educ. 1996:32(2):199-215 https://doi.org/10.1007/BF00138396.

13. Chapell MS, Blanding ZB, Silverstein ME, Takahashi M, Newman B, Gubi A, et al. Test anxiety and academic performance in undergraduate and graduate students. J Educ Psychol. 2005;97(2):268-74 https://doi.org/10.1037/00220663.97.2.268

14. Hembree R. Correlates, causes, effects, and treatment of test anxiety. Rev Educ Res. 1998:58:47-77.

15. Sanford N. Self and society: social change and individual development. New York: Atherton; 1966.

16. Sanford N. Where colleges fail; a study of student as person. San Francisco: JosseyBass; 1986.

17. Shotter J, Gergen KJ. Social construction: knowledge, self, others and continuing the conversation. In: Deetz SA, editor. Communication yearbook. London: Sage; 1994. p. 3-33.

18. Ashmore RD, Deaux K, McLaughlin-Volpe T. An organizing framework for collective identity: articulation and significance of multidimensionality. Psychol Bull. 2004;130(1):80-114 https://doi.org/10.1037/0033-2909.130.1.80.

19. Eisenberg EM. Building a mystery: toward a new theory of communication and identity. J Commun. 2001;51(3):534-52 https://doi.org/10.1111/j.1460-24 66.2001.tb02895.x

20. Poole G. The culturally sculpted self in self-directed learning. Med Educ. 2012:46(8):735-7 https://doi.org/10.1111/j.1365-2923.2012.04312.x.

21. Charmaz K. Constructing grounded theory: a practical guide through qualitative analysis. London: Sage Publications; 2006.

22. Corbin J, Strauss A. Grounded theory research: procedures, canons, and evaluative criteria. Qual Sociol. 1990;13(1):3-21 https://doi.org/10.1007/ BF00988593.

23. Watling CJ, Lingard L. Grounded theory in medical education research: AMEE guide no.70. Med Teach. 2012;34(10):850-61.

24. ScotGEM - Scottish Graduate Entry Medicine. 2020. Retrieved from https:// www.scotlanddeanery.nhs.scot/trainer-information/scottish-graduate-entrymedicine-scotgem/. Accessed on 10 June 2020. 
25. Imperial College London. 2020. Available from: https://www.imperial.ac.uk/ study/ug/courses/school-of-medicine/medicine-graduate-entry/. Accessed on 10 June 2020.

26. Imperial College London MBBS Graduate Course. 2020. Available from: https://www.imperial.ac.uk/study/ug/courses/archived/2018/school-ofmedicine/medicine-graduate-entry/. Accessed on 10 June 2020.

27. Atkinson R, Flint J. Accessing hidden and hard-to-reach populations: snowball research strategies. Soc Res Update. 2001;33(1):1-4 https://sru.soc. surrey.ac.uk/SRU33.PDF

28. Kuzel AJ. Sampling in qualitative inquiry. In: Crabtree BF, Miller WL, editors. Doing qualitative research. Newbury Park: Sage Publications; 1999.

29. Illing J. Thinking about research: theoretical perspectives, ethics and scholarship. In: Swanwick T, editor. Understanding medical education: evidence, theory and practice. The Association for the Study of Medical Education. Chichester: Wiley Blackwell; 2014. p. 371-84.

30. Klag M, Langley A. Approaching the conceptual leap in qualitative research. Int J Manag Rev. 2012;15(2):149-66.

31. Eakin JM, Mykhalovskiy E. Reframing the evaluation of qualitative health research: reflections on a review of appraisal guidelines in the health sciences. J Eval Clin Pract. 2003;9(2):187-94 https://doi.org/10.1046/j.1365-2 753.2003.00392.x.

32. Lincoln YS, Guba EG. Naturalistic inquiry. Newbury Park: Sage Publications; 1985.

33. Denzin NK, Lincolin YS. Strategies of qualitative research. London: Sage Publications; 1998

34. Lewin K. Field theory in social science. New York: Harper; 1951.

35. Flook L, Repetti RL, Ullman JB. Classroom social as predictors of academic performance. Dev Psychol. 2005;41(2):319-27.

36. Angulo-Ruiz F, Pergelova A, Josep R. A market segment approach for higher education based on rational and emotional factors. J Mark High Educ. 2010; 20(1):1-17 https://doi.org/10.1080/08841241003788029.

37. Hemsley-Brown J, Oplatka I. Universities in a competitive global marketplace: a systematic review of the literature on higher education marketing. Int J Public Sect Manag. 2006;19(4):316-38 https://doi.org/10.11 08/09513550610669176.

38. Knowles MS. Self-directed learning. New York: Association Press; 1975.

39. Brockett RG, Hiemstra R. A conceptual frameword for understanind selfdirection in adult learning. In: Self-direction in adult learning: perspectives on theory, research, and practice. London and New York: Routledge; 1991.

40. Ng W. Self-directed learning with web-based sites: how well do students' perceptions and thinking match with their teachers? Teach Sci. 2008;1:2430.

41. Jossberger $H$, Brand-Gruwel $S$, Boshuizen $H$, Wiel M. The challenge of selfdirected and self-regulated learning in vocational education: a theoretical analysis and synthesis of requirements. J Voc Educ Train. 2010;62(4):415-40 https://doi.org/10.1080/13636820.2010.523479.

42. Costello CY. Professional identity crisis: race, class, gender, and success at professional schools. Tennessee: Vanderbilt University Press; 2006. https:// doi.org/10.2307/j.ctv17vf5nn

43. Monrouxe L. Identity, identification and medical education: why should we care? Med Educ. 2009:44:40-9.

44. Murdock JL, Stipanovic N, Lucas K. Fostering connections between graduate students and strengthening professional identity through co-mentoring. $\mathrm{Br}$ J Guid Couns. 2013;41(5):487-503 https://doi.org/10.1080/03069885.2012. 756972.

45. Rees CE. Identities as performances: encouraging visual methodologies in medical education research. Med Educ. 2009;44:5-7.

46. Settles $\mathbb{H}$. When multiple identities interfere: the role of identity centrality. Personal Soc Psychol Bull. 2004;30(4):487-500 https://doi.org/10.1177/01461 67203261885 .

47. Burford B, Rosenthal-Stott HES. First and second year medical students identity and self-stereotype more as doctors than as students: a questionnaire study. BMC Med Educ. 2017;17(1):209 https://doi.org/10.1186/ s12909-017-1049-2

48. Vygotsky L. Interaction between learning and development. Mind Soc. 1978 79-82.

49. Kenny N, Mann K, MacLeod H. Role modelling in physicians' professional formation: reconsidering an essential but untapped educational strategy. Acad Med. 2003;78(12):1203-10 https://doi.org/10.1097/00001888-200312 000-00002.
50. Fahnert B. On your marks, get set, go!-lessons from the UK in enhancing employability of graduates and postgraduates. FEMS Microbiol Lett. 2015; 362(19):1-6.

51. Jelsing EJ, Lachman N, O’Neil AE, Pawlina W. Can a flexible medical curriculum promote student learning and satisfaction? Ann Acad Med Singap. 2007;36(9):713-8.

52. Morgan J, Crooks V, Sampson C, Snyder J. "Location is surprisingly a lot more important than you think": a critical thematic analysis of push and pull factor messaging used on Caribbean offshore medical school websites. BMC Med Educ. 2017;17(1):1-13.

53. Klunklin A, Viseskul N, Sripusanapan A, Turale S. Readiness for selfdirected learning among nursing students in Thailand. Nurs Health Sci. 2010;12(2): 177-81 https://doi.org/10.1111/j.1442-2018.2010.00515.x.

54. Rensburg GH, Botma Y. Bridging the gap between self-directed learning of nurse educators and effective student support. Curationis. 2015:38(2):1-7.

55. O'Shea E. Self-directed learning in nurse education: a review of the literature. J Adv Nurs. 2003;43(1):62-70 https://doi.org/10.1046/j.1365-2648.2 003.02673.x.

56. Shen $\mathrm{W}, \mathrm{Chen} \mathrm{H}, \mathrm{Hu}$ Y. The validity and reliability of the self-directed learning instrument (SDLI) in mainland Chinese nursing students. BMC Med Educ. 2014;14(1):108.

57. McCrorie P. Graduate students are more challenging, demanding, and questioning. BMJ. 2002;10.

58. Hones DF. Known in part: transforming the story, the teller and the narrative researcher: American Educational Research Association; 1997.

59. Yin RK. Applications of case study research. 3rd ed. Los Angeles: Sage Publications; 2012

60. Todd R. An approach to planning organizational transition. Public Adm Rev. 1977;37(5):534-8 https://doi.org/10.2307/974707.

61. Bradford DL. Reinventing organization development: new approaches to change in organizations. San Francisco: Pfeiffer; 2005.

\section{Publisher's Note}

Springer Nature remains neutral with regard to jurisdictional claims in published maps and institutional affiliations.

Ready to submit your research? Choose BMC and benefit from:

- fast, convenient online submission

- thorough peer review by experienced researchers in your field

- rapid publication on acceptance

- support for research data, including large and complex data types

- gold Open Access which fosters wider collaboration and increased citations

- maximum visibility for your research: over $100 \mathrm{M}$ website views per year

At BMC, research is always in progress.

Learn more biomedcentral.com/submissions 